Pacific Journal of 


\section{ON A REPRESENTATION THEORY FOR IDEAL SYSTEMS}

\section{Paul Ezust}

In widely divergent branches of mathematics, objects emerge which bear sufficient formal resemblance to the ideals of rings for them to be called "ideals". In a series of papers, Karl E. Aubert developed an axiomatic theory of ideal systems which subsumes most of the existing "ideal" theories. The goal of this paper is a representation theory for ideal systems in commutative monoids which will allow the formation of a cohomology theory for these systems. One of the results is a theorem which gives at once a monadic (co)homology for each ideal system. The base category in the monad includes PTOP, the category of pointed topological spaces and basepoint-preserving continuous maps, as a full subcategory and, for each ideal system, the category of algebras associated with the monad consists of the module systems over the ideal system. It is the module systems which are the principal objects of this study.

Described below are some of the basic notions of Aubert's theory of ideal systems. For simplicity in connection with our own work we assume that $S$ is a commutative monoid (written multiplicatively) with an annihilating zero element (denoted 0 ).

Definition. A closure operation $x$ on a set $W$ is a function which assigns to each subset $A \subseteq W$ a unique subset $A_{x} \subseteq W$ subject to the following conditions:

(i) $A \subseteq A_{x}$ for all $A \subseteq W$

(ii) $A \subseteq B_{x} \Rightarrow A_{x} \subseteq B_{x}$ for all $A, B \subseteq W$

Note. We do not assume that a closure operation $x$ satisfies the (topological) condition: $(A \cup B)_{x}=A_{x} \cup B_{x}$. In general this condition will not be satisfied.

Definition. A pair $(S, x)$ is an ideal system if $S$ is a commutative monoid with zero and $x$ is a closure operation on $S$ which satisfies the following axioms.

x.1 $\{0\}_{x}=\{0\}$

x.2 $A B_{x} \subseteq B_{x}$ for all $A, B \subseteq S$ ["multiplicative ideal property"]

x.3 $A B_{x} \subseteq(A B)_{x}$ for all $A, B \subseteq S$ ["continuity axiom"]. 
Terminology. The sets $A_{x} \subseteq S$ are called the $x$-ideals of $S$.

Notation. $A+B=(A \cup B)_{x}$

$$
\begin{aligned}
& A: B=\{s \in S \mid s b \in A \quad \forall b \in B\} \\
& a \equiv b\left(A_{x}\right) \text { iff } A_{x}+\{a\}=A_{x}+\{b\} .
\end{aligned}
$$

Several examples of particular ideal systems are discussed in Aubert's extensive survey paper [2] and the reader is referred to that paper for definitions, etc.

In a brief note [8], Aubert and Hansen introduced the notion of "module system" over an ideal system as an ancillary device to the theory of ideal systems. Despite the pessimism expressed in that paper, it is our purpose to show that the theory of module systems over ideal systems yields a representation theory analogous to the theory of modules over rings.

Throughout this paper the terminology and notations of category theory have been used as are found in such standard texts as Herrlich and Strecker [14] and Mitchell [22]. The author originally became interested in the problems discussed herein during a course given by Professor Karl E. Aubert at Tufts University during the academic year, 1969-70.

\section{Axioms for module-systems.}

Definition. Let $(S, x)$ be a fixed ideal system. A left $S$-set is a set $M$ together with a map $S \times M \rightarrow M$, denoted by $(s, m) \rightarrow s m$, satisfying

(i) $s(t m)=(s t) m \forall s, t \in S, \forall m \in M$

(ii) $1 m=m \forall m \in M$ (where 1 denotes the identity element of the monoid $S$ ).

Definition. A pair $(M, y)$, where $M$ is a (left) $S$-set and $y$ is a closure operation on $M$, is a module-system over $(S, x)$ if the following are satisfied:

y.1 $\exists \theta \in M$ such that $0 m=\theta \forall m \in M$, and $\{\theta\}_{y}=\{\theta\}$. We shall denote $\theta=0$.

y.2 $A U_{y} \subseteq U_{y} \quad \forall A \subseteq S, \forall U \subseteq M$

y.3 $A U_{y} \subseteq(A U)_{y} \quad \forall A \subseteq S, \forall U \subseteq M$

y.4 $A_{x} U \subseteq(A U)_{y} \forall A \subseteq S, \forall U \subseteq M$.

Notation. Let $(M, y)$ be a module-system, let $U, V \subseteq M, A \subseteq S$, $u, v, w \in M$, and $s \in S$. Then,

$$
\begin{aligned}
& U+V=(U \cup V)_{y} \\
& U: V=\{s \in S \mid s v \in U \forall v \in V\}
\end{aligned}
$$




$$
\begin{aligned}
& U: A=\{m \in M \mid a m \in U \forall a \in A\} \\
& \operatorname{Ann}(u)=0: u(=\{0\}:\{u\}) \text { in } S \\
& \operatorname{Ann}(a)=0: a(=\{0\}:\{a\}) \text { in } M \\
& w \equiv v\left(U_{y}\right) \text { iff } U_{y}+\{w\}=U_{y}+\{v\} .
\end{aligned}
$$

Observation. Frequent use shall be made of the following two equivalences which were established by Aubert and Hansen [8].

1. Axiom y. 3 is equivalent to the following statement:

$$
\left(U_{y}: s\right)_{y}=U_{y}: s \quad \forall U \subseteq M, \forall s \in S .
$$

2. Axiom y.4 is equivalent to the following statement:

$$
\left(U_{y}: v\right)_{x}=U_{y}: v \quad \forall U \subseteq M, \forall v \in M .
$$

Definition. Let $(S, x)$ be a fixed ideal system. The category $M S$ consists of objects which are module systems $(M, y)$ over $(S, x)$ and morphisms $\xi:\left(M_{1}, y_{1}\right) \rightarrow\left(M_{2}, y_{2}\right)$ which are set functions that satisfy the following conditions:

(i) $\xi(s u)=s \xi(u) \forall s \in S, \forall u \in M_{1}$

(ii) $\xi\left(U_{y_{1}}\right) \subseteq(\xi(U))_{y_{2}} \forall U \subseteq M_{1}$.

Remark. Morphism condition (ii), above, is equivalent to:

$$
\left(\xi^{-1}\left(V_{y_{2}}\right)\right)_{y_{1}}=\xi^{-1}\left(V_{y_{2}}\right) \forall V \subseteq M_{1}
$$

EXAmples.

1. For any fixed ideal system $(S, x)$, let $M=A_{x}$ for some $A \subseteq S$, and $y=x$. Thus, for $B \subseteq M, B_{y}=B_{x}$, and $(M, y)$ is an object of $M S$.

2. Let $S$ be the multiplicative semigroup of a commutative ring with identity, and let $x$ be the classical ideal closure, $A_{x}=A_{d}=\langle A\rangle$ $\forall A \subseteq S$. Then any module $M$ over the ring, with the classical submodule closure, $U_{y}=\langle U\rangle$, is an object of $M S$.

3. Let $S$ be a commutative monoid with 0 and for each $A \subseteq S$, let $A_{x}=S A$ [this closure is called the $s$-closure]. For any $S$-set, $M$, and any $U \subseteq M$, define $U_{y}=S U$ [this closure will be referred to as the $s$-closure also]. Then $(M, y)$ is an object of $M S$.

4. Let $(S, x)$ be an ideal system and let $M$ be an $S$-set. For any $U \subseteq M$, define $U_{y}=U \cup\{0\}$ [this closure will be referred to as the discrete closure on $M]$. Then $(M, y)$ is an object of $M S$.

5. Let $S=\{1 / n \mid n \in Z, n>0\} \cup\{0\}$. For each $A \subseteq S$, define $A_{x}=\{s \in S \mid s \leqq \sup A\}$; i.e., $A_{x}=[0, \bar{a}]$, where $\bar{a}=\sup A$. Then 
$(S, x)$ is an example of an ideal system for which the inclusion $x .3$ is proper.

\section{The morphisms of $M S$.}

Definition. An $S$-set $M$ with 0 is called an $(S, x)$-set provided $(0: u)_{x}=0: u$ for all $u \in M$. A map $\varphi: M_{1} \rightarrow M_{2}$ from one $S$-set to another is called an $S$-map if it satisfies (i) above.

Proposition 1. Let $M$ be an $(S, x)$-set and $\left\{f_{j}: M \rightarrow M_{j} \mid j \in J\right\} a$ family of $S$-maps, where $\left\{\left(M_{j}, y_{j}\right) \mid j \in J\right\}$ is a family of objects of $S_{I}$. Then there exists a closure operator $y$ such that $(M, y)$ is an object and $f_{l}: M \rightarrow M_{j}$ is a morphism for all $j \in J$. The coarsest such system $y$ is said to be induced in $M$ by the family $\left\{f_{j}: M \rightarrow M_{j} \mid j \in J\right\}$.

Proof. Let $M$ be an $(S, x)$-set and $F=\left\{f_{j}: M \rightarrow M_{j} \mid j \in J\right\}$ be a family of $S$-maps into objects $\left(M_{j}, y_{j}\right)$, for $j \in J$. Let $Q=\left\{f_{j}^{-1}\left(U_{y_{j}}^{j}\right)\right\}$ $\left.U^{j} \subseteq M_{j}, \quad j \in J\right\} \cup\{0\} . \quad$ For any $V \subseteq M$ define $V_{y}=\cap\{W \in Q \mid$ $V \subseteq W\}$.

Definition. Let $M$ be an $(S, x)$-set and $G=\left\{g_{j}: M_{j} \rightarrow M \mid j \in J\right\}$ be a family of $S$-maps from objects $\left(M_{j}, y_{j}\right)$ to $M$. The finest closure system, $y$, on $M$ (if one exists) such that $(M, y)$ is an object of $M S$ and such that each $g_{j}$ is a morphism, will be called the closure system which is coinduced in $M$ by the family $G$. Let $P=\left\{U \subseteq M \mid\left(g_{j}^{-1}(U)\right)_{y_{j}}=g_{j}^{-1}(U)\right.$ for all $j \in J\} . \quad G$ is called a covering family of $S$-maps into $M$ if (1) for each $U \in P, \exists j \in J$ such that $g_{\jmath}\left(g_{j}^{-1}(U)\right)=U$; and (2) $0 \in P$.

Proposition 2. Let $M$ be an $(S, x)$-set and let $G=\left\{g_{j}: M_{j} \rightarrow M \mid\right.$ $j \in J\}$ be a covering family of $S$-maps from objects $\left(M_{j}, y_{j}\right)$ to $M$. Then there exists a coinduced closure system y for $M$ (with respect to $G$ ).

Proof. Let $M$ and $G$ be as described above and let $P$ be as defined above. Let $Q=\left\{U \in P \mid(U: m)_{x}=U: m \forall m \in M\right\}$ and, for each $V \subseteq M$, define $V_{y}=\cap\{U \in Q \mid V \subseteq U\}$

Definition. An equivalence relation $\sim$ on an object $(M, y)$ is a congruence if $u \sim v \Rightarrow s u \sim s v \forall s \in S$. Let $[v]=\{u \in M \mid u \sim v\} . \quad$ A congruence $\sim$ is admissible if $[0]_{y}=[0]$.

Proposition 3. Let $(M, y)$ be an object of $M S$ and $\sim$ an admissible congruence on $M$. Then $(M / \sim, \tilde{y})$ is an object of $M S$, where $M / \sim$ is 
the set of $\sim$ classes in $M$ and $\tilde{y}$ is coinduced by the map $\pi: M \rightarrow M / \sim$ defined by $\pi(u)=[u] \forall u \in M$.

Proof. By Proposition 2, one need only show that for any admissible congruence $\sim$ on an object $(M, y)$, the set $M / \sim$ is an $(S, x)$-set and the map $\pi: M \rightarrow M / \sim$ is a covering $S$-map.

Proposition 4. Let $(M, y)$ be an object and $U_{y} \subseteq M$. Then

(a) $U_{y}$ determines an admissible congruence on M'given by the rule: $u \equiv v\left(U_{y}\right)$ iff $U_{y}+\{u\}=U_{y}+\{v\}$. Denote the set of congruence classes "modulo $U_{y}$ " by $M / U_{y}$.

(b) The inclusion map i: $U_{y} \rightarrow M$ induces a system $y^{\prime}$ on $U_{y}$ given by the rule: $V_{y^{\prime}}=V_{y} \cap U_{y}=V_{y} \forall V \subseteq U_{y}$. Thus, $U_{y}$ is a subobject of $M$. The prime is generally omitted.

Proposition 5. The Zero object, $M=\{0\}$, is both initial and terminal in MS and will be denoted, simply, 0.

TheOrem 6. Let $\varphi:\left(M_{1}, y_{1}\right) \rightarrow\left(M_{2}, y_{2}\right)$ be a morphism. Then

(a) $\varphi$ is a monomorphism iff $\varphi$ is injective.

(b) $\varphi$ is an epimorphism iff $\varphi$ is surjective.

(c) If $\varphi$ is monic then $\left(\varphi^{-1}(U)\right)_{y_{1}} \subseteq \varphi^{-1}\left(U_{y_{2}}\right) \forall U \subseteq M_{2}$.

Proof. (a) Suppose $\varphi$ is a monomorphism such that $\varphi(u)=\varphi(v)$ for some $u, v \in M_{1}$. Define $\left(M_{3}, y_{3}\right)$ by: $M_{3}=S \vee S$, the disjoint union of two copies of $S$ (labeled with $u$ and $v$, respectively) with the zero elements identified, and $U_{y_{3}}=\left(U \cap S_{u}\right)_{x} \cup\left(U \cap S_{v}\right)_{x} \forall U \subseteq M_{3}$. In fact, this construction is a special case of the more general construction of the coproduct of $S$ with itself, which is discussed in $\S 4$. Let $\psi_{1}: M_{3} \rightarrow M_{1}$ be defined by the rule: $\psi_{1}\left(s_{u}\right)=s u$ and $\psi_{1}\left(s_{v}\right)=s v \quad \forall s \in S$. Define $\psi_{2}: M_{3} \rightarrow M_{1}$ by the rule: $\psi_{2}\left(s_{u}\right)=s v$ and $\psi_{2}\left(s_{v}\right)=s u \forall s \in S . \quad \psi_{1}$ and $\psi_{2}$ are morphisms such that $\varphi \psi_{1}=\varphi \psi_{2}$. Since $\varphi$ is monic, it follows that $\psi_{1}=\psi_{2}$; i.e., $u=v$.

(b) Suppose $\varphi$ is an epimorphism. Then $\varphi\left(M_{1}\right)$ is an $S$-set.

Claim. $\varphi\left(M_{1}\right)=M_{2}$. Let $M_{3}=M_{2} / \varphi\left(M_{1}\right)$ be the $S$-set of congruence classes in $M_{2}$ modulo the $S$-set $\varphi\left(M_{1}\right)$; i.e., for $u, v \in M_{2}, u \equiv$ $v\left(\varphi\left(M_{1}\right)\right)$ means $S u \cup \varphi\left(M_{1}\right)=S v \cup \varphi\left(M_{1}\right)$. For any $U \subseteq M_{3}$, define $U_{y_{3}}=S U$. Let $\pi: M_{2} \rightarrow M_{3}$ be the $S$-map $\pi(u)=[u]$ and let $M=$ $\left\{(u,[u]) \mid u \in M_{2}\right\} \cup\left\{(u,[0]) \mid u \in M_{2}\right\}$. For each $s \in S, s(u,[u])=$ $(s u, s[u])=(s u,[s u])$ and $s(u,[0])=(s u,[0])$. Also, for each $u \in M_{2}$, $s(u,[0])=(0,[0])$ iff $s u=0$ and $s(u,[u])=(0,[0])$ iff $s u=0$, so that $(0,[0]):(u,[0])=0: u=(0: u)_{x}$. Hence, $M$ is an $(S, x)$-set. Define $\xi_{1}: M_{2} \rightarrow M$ by the rule: $\xi_{1}(u)=(u,[u]) \forall u \in M_{2}$. Define $\xi_{2}: M_{2} \rightarrow M$ 
by the rule: $\xi_{2}(u)=(u,[0]) \forall u \in M_{2}$. Then $\left\{\xi_{1}, \xi_{2}\right\}$ is a covering family of $S$-maps into $M$. Let $y$ be coinduced on $M$ by $\left\{\xi_{1}, \xi_{2}\right\}$ and note that $\xi_{1} \varphi=\xi_{2} \varphi$; hence, $\xi_{1}=\xi_{2}$. Thus, $\pi(u)=[0] \forall u \in M_{2}$; i.e., $\varphi\left(M_{1}\right)=M_{2}$.

(c) Suppose $\varphi$ is a monomorphism. Then, by (a) above, $\varphi$ is injective. Thus, $\varphi\left(\left(\varphi^{-1}(U)\right)_{y_{1}}\right) \subseteq U_{y_{2}}$; hence, $\left(\varphi^{-1}(U)\right)_{y_{1}} \subseteq \varphi^{-1}\left(U_{y_{2}}\right)$.

Theorem 7. MS has (a) Kernels, (b) Images, (c) Cokernels, and (d) Coimages.

Proof. Let $\varphi:\left(M_{1}, y_{1}\right) \rightarrow\left(M_{2}, y_{2}\right)$ be a morphism. (a) $\operatorname{Ker} \varphi=$ $\varphi^{-1}(0)$. (b) $\operatorname{Im} \varphi=\left(\varphi\left(M_{1}\right), y \varphi\right)$, where the closure operator $y \varphi$ is coinduced by the (surjective) map $\varphi^{\prime}: M_{1} \rightarrow \varphi\left(M_{1}\right)$ defined by the rule: $\varphi^{\prime}(u)=\varphi(u) \forall u \in M_{1}$. (c) Define the congruence $\sim$ by the rule: $u \sim u$ $\forall u \in M_{2}$ and, for $u \neq v, u \sim v$ iff $\{u, v\} \subseteq\left(\varphi\left(M_{1}\right)\right)_{y_{2}}$. In forming $M_{2} / \sim$, the $S$-set of $\sim$ classes, $\left(\varphi\left(M_{1}\right)\right)_{y_{2}}$ is compressed down to [0] and the rest of $M_{2}$ remains unchanged. Let $\pi: M_{2} \rightarrow M_{2} / \sim$ be the projection $u \rightarrow[u]$. Note that $[u]=[0]$ for $u \in\left(\varphi\left(M_{1}\right)\right)_{y_{2}}$ and $[u]=u$ for $u \notin\left(\varphi\left(M_{1}\right)\right)_{y_{2}}$. Also note that $M_{2} / \sim$ is an $(S, x)$-set and let $\tilde{y}$ be coinduced by $\{\pi\}$. Then Coker $\varphi=\left(M_{2} / \sim, \tilde{y}\right)$. (d) For each $u \in M_{1}$, let $\bar{u}=\varphi^{-1}(\varphi(u))$ and let $M_{1} / \varphi=\left\{\bar{u} \mid u \in M_{1}\right\}$. Let $\pi: M_{1} \rightarrow M_{1} / \varphi$ be the projection, $u \rightarrow \bar{u}$. For each subset $\pi(U) \subseteq M_{1} / \varphi$, define $(\pi(U))_{\varphi y}=$ $\hat{\varphi}^{-1}\left((\varphi(U))_{y_{2}}\right)$, where $\hat{\varphi}: M_{1} / \varphi \rightarrow M_{2}$ is the map, $\hat{\varphi}(\bar{u})=\varphi(u)$, for all $\bar{u} \in M_{1} / \varphi$. Then $\operatorname{Coim} \varphi=\left(M_{1} / \varphi, \varphi y\right)$.

Remarks. (1) $\varphi$ monic $\Rightarrow \operatorname{Im} \varphi \cong M_{1}$.

(2) In any exact category (e.g., the category of modules over a commutative ring with unity), for any morphism $\varphi: M_{1} \rightarrow M_{2}, \operatorname{Im} \varphi \cong$ $\operatorname{Coim} \varphi$. The following example shows that this is not generally true in MS.

ExAmple. Let $M=\{0, a, b, c\}, \quad S=\{0,1\}$, with the obvious multiplication. Let $\left(M_{1}, y_{1}\right)$ and $\left(M_{2}, y_{2}\right)$ be defined as follows. $M_{1}=$ $M_{2}=M . \quad y_{1}$ is the $s$-system on $M_{1}$, and $y_{2}$ is the indiscrete system on $M_{2}$ : $\{0\}_{y_{2}}=\{0\}$, and $U \neq\{0\} \Rightarrow U_{y_{2}}=M_{2}$. Let $\varphi: M_{1} \rightarrow M_{2}$ be the identity map. Then $\left(M_{2}, y \varphi\right)=\operatorname{Im} \varphi \not \operatorname{Coim} \varphi=\left(M_{1}, \varphi y\right)$.

PROPOSITION 8. Let $\varphi: M_{1} \rightarrow M_{2}$ be a morphism. If $\varphi\left(U_{y_{1}}\right)=$ $(\varphi(U))_{y_{2}}$ for all $U \subseteq M_{1}$ then $\operatorname{Im} \varphi \cong \operatorname{Coim} \varphi$.

Observation. The example which precedes Proposition 8 also illustrates the fact that a morphism in $M S$ might be both monic and epic and yet fail to be an isomorphism; i.e., $M S$ is not balanced. Another way of characterizing this situation is to note that the forgetful functor $F: M S \rightarrow S E T$ does not reflect isomorphisms. It follows (Proposition 32.5 [14]) that $M S$ is not an algebraic category. 


\section{Categorical constructions in $M S$.}

\section{Theorem 9. MS has Products.}

Proof. Let $\left\{\left(M_{j}, y_{j}\right) \mid j \in J\right\}$ be a family of objects of $M S$. Let $\Pi M_{j}$ denote the cartesian product of the sets $M_{i}(j \in J)$. For each $\left(m_{l}\right) \in \Pi M_{j}$ and each $s \in S$, define $s\left(m_{j}\right)=\left(s m_{j}\right)$. Let 0 denote $\left(0_{j}\right)$ and observe that, for all $\left(m_{j}\right) \in \Pi M_{j}, 0:\left(m_{l}\right)=\cap\left\{0: m_{j} \mid j \in J\right\}$, the latter being an intersection of $x$-ideals in $S$. Thus, $\Pi M_{j}$, is an $(S, x)$-set. For each $k \in J$, define $\pi_{k}: \Pi M_{j} \rightarrow M_{k}$ by the rule, $\pi_{k}\left(\left(m_{j}\right)\right)=m_{k}$ (this is the canonical projection map from the cartesian product to its factors). Let $\Pi y_{j}$ be the system induced in $\Pi M_{j}$ by the family of projections, $\left\{\pi_{j} \mid j \in J\right\}$. Then, for each $U \subseteq \Pi M_{j}, U_{\text {II } y_{j}}=\cap\left\{\pi_{j}^{-1}\left(\left(\pi_{j}(U)\right)_{y_{j}}\right) \mid j \in J\right\}=\times\left\{\left(\pi_{j}(U)\right)_{y_{j}} \mid j \in J\right\} . \quad$ It is easy to verify that $\left(\Pi M_{i}, \Pi y_{j}\right)$ is the product.

Notation. $\quad M_{1} \times M_{2}$ will frequently be used to denote the product, $\Pi\left\{M_{l} \mid j=1,2\right\}$, of two objects of $M S$. The corresponding closure system will be denoted, $y_{1} \times y_{2}$.

\section{THEOREM 10. MS has Coproducts.}

Proof. Let $\left\{\left(M_{j}, y_{j}\right) \mid j \in J\right\}$ be a family of objects of $M S$ and let $\Sigma M_{j}$ denote the disjoint union, $\vee\left\{M_{j} \mid j \in J\right\}$ with all zeros identified. For each $k \in J$, let $\delta_{k}: M_{k} \rightarrow \Sigma M_{j}$ be the natural inclusion map. Let $\Sigma y_{j}$ be defined on $\Sigma M_{j}$ as follows. For any $U \subseteq \Sigma M_{j}, U_{\Sigma y_{j}}=\vee\left\{\left(\delta_{l}^{-1}(U)\right)_{y_{k}} \mid\right.$ $k \in J\}$. Note that $U_{\Sigma y_{j}}=\cup\left\{\left(U \cap M_{k}\right)_{y_{k}} \mid k \in J\right\}$ if we identify $M_{k}$ with its set-theoretic image, $\delta_{k}\left(M_{k}\right)$ in $\Sigma M_{j}$. Clearly $\left(\Sigma M_{j}, \Sigma y_{l}\right)$ is an object of $M S$ and each map $\delta_{k}$ is a morphism. Note that $\Sigma y_{j}$ is the closure system coinduced in $\Sigma M_{j}$ by the family of inclusions, $\left\{\delta_{l} \mid j \in J\right\}$. It is not hard to verify that $\left(\Sigma M_{j}, \Sigma y_{j}\right)$ is the coproduct.

Definition. An object of $M S$ is free if it is of the form $\Sigma M_{j}(j \in J)$, where for each $j \in J,\left(M_{l}, y_{l}\right) \cong(S, x)$. We denote such an object $(F(J)$, $\left.y_{*}\right)$ and refer to the index set $J$ as the basis for the free object $\left(F(J), y_{*}\right)$.

REMARK. In particular, $(S, x)$ is free with basis $\{1\}$.

Proposition 11. [Universal Mapping Property of Free Objects]. $\left(F(U), y_{*}\right)$ is a free object with basis $U$ iff for any object $(M, y)$ and any set map $\sigma: U \rightarrow M$, there is a unique morphism $\varphi: F(U) \rightarrow M$ such that $\varphi \eta=\sigma$, where $\eta: U \rightarrow F(U)$ is the inclusion, $u \rightarrow 1_{u}$ for all $u \in U$.

Definition. The morphism $\varphi$ described above is called the lift of $\sigma$. 
Proposition 12. Let $(M, y)$ be an object of $M S$ and let $\varphi: F(M) \rightarrow M$ be the epimorphism that lifts the identity morphism 1: $M \rightarrow M$. Then $M \cong \operatorname{Coim}(\varphi)$.

Definition. An object $(M, y)$ is projective if for any morphism $\theta: M \rightarrow M_{2}$ and any epimorphism $\psi: M_{1} \rightarrow M_{2}$ [where $\left(M_{1}, y_{1}\right)$ and $\left(M_{2}, y_{2}\right)$ are objects] there exists a morphism $\xi: M \rightarrow M_{1}$ such that $\psi \xi=\theta$.

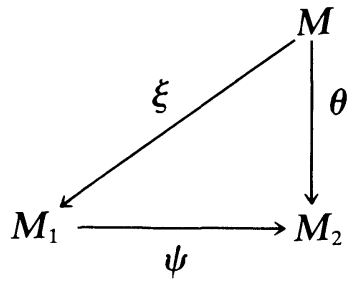

REMARK. It follows immediately from the above definition that if $(M, y)$ is projective, then $\left(M, y^{\prime}\right)$ is projective for any closure system $y^{\prime}$ (on $M$ ) which is finer than $y$. Thus, since the $s$-system is the finest possible closure system for $M$, each projective object in the category $E N S$ - $S$ of all $S$-sets determines a family of projective objects of $M S$ and, conversely, each projective object of $M S$ determines a projective object of $E N S-S$.

Proposition 13. Let $(M, y)$ be an object of $M S$. Then $M$ is projective iff $M$ is a retract of a free object of $M S$ [In particular, each free object of $M S$ is projective.]

Remark. In the category $R$-Mod, of left $R$-modules, an object is a retract of a free iff it is a direct summand of a free. The following example demonstrates that this is not the case in general in MS.

EXAMPLE. Let $S=\{0,1, a, b\}$ with multiplication defined as follows: $a a=b b=a b=b a=a$. Let $M=\{0, a\}$ and let $S$ and $M$ each have the $s$-system closure. Then $(M, y)$ is a projective object of $M S$ and $M$ is not a direct summand of $S$ since $(S-M)_{y} \neq(S-M) \cup\{0\}$. Since a free object of $M S$ must be a coproduct of copies of $S$ it follows that $M$ is not a direct summand of any free object.

Proposition 14. Let $\left\{\left(M_{j}, y_{j}\right) \mid j \in J\right\}$ be a family of objects of MS. Then $\left(\Sigma M_{j}, \Sigma y_{j}\right)$ is projective iff $\left(M_{j}, y_{j}\right)$ is projective $\forall j \in J$.

REMARK. In view of Theorems 9 and 10 , it is clear that $M S$ is not an additive category since finite products are not isomorphic to finite coproducts. 


\section{Completeness and cocompleteness of $M S$.}

Proposition 15. MS is locally and colocally small.

PROPOSITION 16. MS has Intersections.

Proof. Let $\left\{\alpha_{j}:\left(M_{j}, y_{j}\right) \rightarrow(M, y) \mid j \in J\right\}$ be a family of subobjects of $(M, y)$. Since $\alpha_{j}$ monic $\Rightarrow M_{j} \cong \operatorname{Im} \alpha_{j}$, for each $j \in J$ we take $M^{\prime}=$ $\cap\left\{\operatorname{Im} \alpha_{J} \mid j \in J\right\}$, a set-theoretic intersection of subsets of $M$. For each $j \in J$, let $\beta_{j}: M^{\prime} \rightarrow \operatorname{Im} \alpha_{j}$ be the natural inclusion map. Then $M^{\prime}$ is an $(S, x)$-set and $\beta_{j}$ is an $S$-map for each $j \in J$. Let $y^{\prime}$ be the system induced on $M^{\prime}$ by the family $\left\{\beta_{j} \mid j \in J\right\}$. Let $\alpha: M^{\prime} \rightarrow M$ be the natural inclusion map. Then $\alpha:\left(M^{\prime}, y^{\prime}\right) \rightarrow(M, y)$ is the intersection of the subobjects $\left\{\alpha_{j} \mid j \in J\right\}$.

\section{Proposition 17. MS has Equalizers.}

Proof. Let $\varphi, \theta: M_{1} \rightarrow M_{2}$ be morphisms, and let $E=\left\{u \in M_{1} \mid\right.$ $\varphi(u)=\theta(u)\}$. Then $\operatorname{Equ}(\varphi, \theta)=\left(E, y_{e}\right)$, where $y_{e}$ is induced by the inclusion $\eta: E \rightarrow M_{1}$.

The following Theorem follows from Theorem 23.8 [14].

THEOREM 18. MS has the following properties:

(a) MS is complete (in particular, MS has inverse limits).

(b) MS has (multiple) pullbacks.

(c) MS has inverse images.

From Theorems 10 and 18 and Proposition 15 we obtain the hypotheses of Theorem 23.12 [14], and using the dual of Theorem 23.8 [14] we obtain the following

THEOREM 19. MS has the following properties:

(a) $M S$ is cocomplete (in particular, $M S$ has direct limits).

(b) MS has (multiple) pushouts.

(c) MS has direct images.

(d) MS has coequalizers.

(e) MS has cointersections.

\section{Properties of the hom functor $M S \rightarrow M S$.}

THEOREM 20. For each pair of objects $\left(M_{1}, y_{1}\right),\left(M_{2}, y_{2}\right)$ of $M S$, $\left(\operatorname{hom}_{s}\left(M_{1}, M_{2}\right), \hat{y}\right)$ is an object of $M S$, where, for $\varphi \in \operatorname{hom}_{s}\left(M_{1}, M_{2}\right)$ and $s \in S, s \varphi$ is defined by the rule: $(s \varphi)(u)=s(\varphi(u)) \forall u \in M_{1}$, and, for any 
$W \subseteq \operatorname{hom}_{s}\left(M_{1}, M_{2}\right), W_{\hat{y}}=\cap\left\{\left[m, U_{y_{2}}\right] \mid W \subseteq\left[m, U_{y_{2}}\right]\right\}$, where $\left[m, U_{y_{2}}\right]=$ $\left\{\xi \in \operatorname{hom}_{s}\left(M_{1}, M_{2}\right) \mid \xi(m) \in U_{y_{2}}\right\}$.

Proposition 21. For any object $(M, y)$ of $M S,(M, y) \cong$ $\left(\operatorname{hom}_{s}(S, M), \hat{y}\right)$, where $(S, x)$ is considered as an object of $M S$.

THEOREM 2.2. MS has an internal Hom functor, Hom: $M S^{o p} \times$ $M S \rightarrow M S$.

Proof. By Theorem 20 it will suffice to verify that $\operatorname{hom}_{s}(\varphi, \theta): \operatorname{hom}_{s}\left(M_{1}, M_{2}\right) \rightarrow \operatorname{hom}_{s}\left(M_{1}^{\prime}, M_{2}^{\prime}\right)$ is a morphism for all $\varphi \in$ $\operatorname{hom}_{s}\left(M_{1}^{\prime}, M_{1}\right)$ and all $\theta \in \operatorname{hom}_{s}\left(M_{2}, M_{2}^{\prime}\right)$.
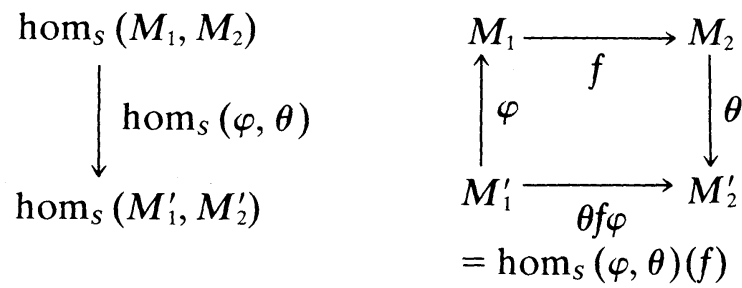

Indeed, it is true in any category that the corresponding construction yields a well defined set map. Thus, with $\operatorname{hom}_{s}(\varphi, \theta)(f)=\theta f \varphi$, we have the following equations:

$$
\begin{aligned}
\operatorname{hom}_{S}(\varphi, \theta)^{-1}\left(\left[u, U_{y^{2}}\right]\right)=\left\{f \in \operatorname{hom}_{S}\left(M_{1}, M_{2}\right) \mid \theta f \varphi(u) \in U_{y^{2}}\right\} \\
=\left\{f \in \operatorname{hom}_{S}\left(M_{1}, M_{2}\right) \mid f(\varphi(u)) \in \theta^{-1}\left(U_{y^{\prime}}\right)\right\}=\left[\varphi(u), \theta^{-1}\left(U_{y^{2}}\right)\right] .
\end{aligned}
$$

Notation. Since $M S$ has an internal Hom functor, we will follow the practice of Herrlich and Strecker [14] and others and write it with a capital $H$. Also, we will suppress the subscript $S$ when no confusion will result.

Proposition 23. For any family $\left\{\left(M_{j}, y_{j}\right) \mid j \in J\right\}$ of objects of $M S$, $\operatorname{Hom}\left(\Sigma M_{j}, M\right) \cong \Pi \operatorname{Hom}\left(M_{j}, M\right)$ for any object $(M, y)$.

Proposition 24. The functor $\operatorname{Hom}\left(M,{ }_{-}\right): M S \rightarrow M S$ (for fixed object $(M, y))$ preserves products; i.e., for any family $\left\{\left(M_{j}, y_{j}\right) \mid j \in J\right\}$ of objects, $\operatorname{Hom}\left(M, \Pi M_{j}\right) \cong \Pi \operatorname{Hom}\left(M, M_{l}\right)$.

Proposition 25. The functor $\operatorname{Hom}\left(M,_{-}\right): M S \rightarrow M S$ preserves equalizers.

Proof. Let $f, g \in \operatorname{Hom}\left(M_{1}, M_{2}\right)$. 

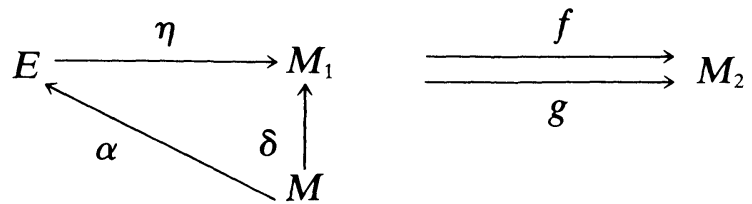

Then, by Prop. I.17, $\eta: E \rightarrow M_{1}$ is the equalizer of $f$ and $g$, where $E=\left\{u \in M_{1} \mid f(u)=g(u)\right\}$ and $y_{e}$, the closure on $E$, is induced by the canonical inclusion, $\eta: E \rightarrow M_{1}$.

To prove that $\operatorname{Hom}\left(M,{ }_{-}\right)$preserves equalizers we shall show that $\operatorname{Hom}(M, E) \cong \operatorname{Equ}(\hat{f}, \hat{g})$, where $\hat{f}=\operatorname{Hom}(M, f)$ and $\hat{g}=\operatorname{Hom}(M, g)$, and $\operatorname{Equ}(\hat{f}, \hat{g})=\left\{\xi \in \operatorname{Hom}\left(M, M_{1}\right) \mid \hat{f}(\xi)=\hat{g}(\xi)\right\}$.
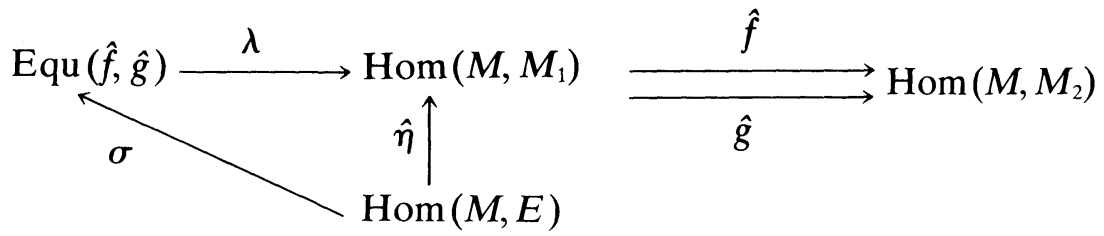

Let $\lambda: \operatorname{Equ}(f, g) \rightarrow \operatorname{Hom}\left(M, M_{1}\right)$ be the canonical inclusion and $\hat{\eta}=\operatorname{Hom}(M, \eta): \operatorname{Hom}(M, E) \rightarrow \operatorname{Hom}\left(M, M_{1}\right) ;$ i.e., $\hat{\eta}(k)=\eta k$. Then $\hat{f} \hat{\eta}=\hat{g} \hat{\eta}$, hence there exists a morphism, $\sigma: \operatorname{Hom}(M, E) \rightarrow \operatorname{Equ}(\hat{f}, \hat{g})$ such that $\hat{\eta}=\lambda \sigma . \quad \sigma$ is the required isomorphism.

The next Proposition follows from Theorem 24.3 [14].

Proposition 26. $\operatorname{Hom}\left(M, \_\right): M S \rightarrow M S$ preserves pullbacks, multiple pullbacks, terminal objects, inverse images, finite intersections, and limits.

THEOREM 27. $\operatorname{Hom}\left(M,{ }_{-}\right): M S \rightarrow M S$ has a left adjoint.

Proof. Consider the functor diagram, where $G=\operatorname{Hom}\left(M,{ }_{-}\right)$, $U=\operatorname{hom}_{s}\left(M,{ }_{-}\right)$, and $V=$ Forgetful.

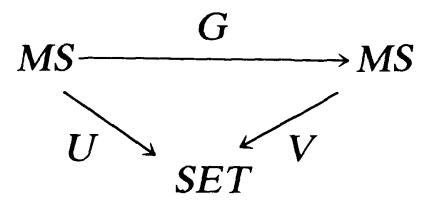

Clearly this diagram commutes. By Propositions 15, 18, and 19, MS is complete, cocomplete, locally small, and colocally small. By Proposition 26, $G$ preserves limits. By Theorem 30.20 [14], $U$ has a left adjoint. Clearly $V$ is faithful. The result follows from Theorem 28.12 [14]. 


\section{The tensor product in $M S$.}

Definition. We denote the left adjoint of $\operatorname{Hom}\left(M,{ }_{-}\right): M S \rightarrow M S$ - $\otimes M$, and we refer to $M^{\prime} \otimes M$ as the tensor product of $M^{\prime}$ and $M$. The closure system on $M^{\prime} \otimes M$ is denoted $y^{\prime} \otimes y$.

REMARKs. The adjoint situation, $(\eta, \delta):_{-} \otimes M \dashv \operatorname{Hom}\left(M,{ }_{-}\right)$, gives, for each object $M_{1}$ of $M S$ a morphism $\eta_{M_{1}}: M_{1} \rightarrow \operatorname{Hom}\left(M_{2}, M_{1} \otimes M_{2}\right)$. Define $\psi: M_{1} \times M_{2} \rightarrow M_{1} \otimes M_{2}$ by the rule, $\psi\left(\left(u_{1}, u_{2}\right)\right)=\left(\eta_{M_{1}}\left(u_{1}\right)\right)\left(u_{2}\right)$ and denote $\psi\left(\left(u_{1}, u_{2}\right)\right)=u_{1} \otimes u_{2}$. Note that $s\left(u_{1} \otimes u_{2}\right)=s u_{1} \otimes u_{2}=u_{1} \otimes s u_{2}$, for all $s \in S$. In fact, $\psi$ is bilinear, in the sense that both $\psi\left(u_{1},-\right): M_{2} \rightarrow M_{1} \otimes M_{2}$ and $\psi\left({ }_{-}, u_{2}\right)$ : $M_{1} \rightarrow M_{1} \otimes M_{2}$ are morphisms (defined in the obvious ways). Indeed, $\psi\left(u_{1},{ }_{-}\right)=\eta_{M_{1}}\left(u_{1}\right) \in \operatorname{Hom}\left(M_{2}, M_{1} \otimes M_{2}\right)$ by definition. To see that $\psi\left({ }_{-}, u_{2}\right) \in \operatorname{Hom}\left(M_{1}, M_{1} \otimes M_{2}\right)$, note that

$$
\begin{aligned}
\psi\left({ }_{-}, u_{2}\right)^{-1}\left(U_{y_{1} \otimes y_{2}}\right) & =\left\{u_{1} \in M_{1} \mid \psi\left(u_{1}, u_{2}\right) \in U_{y_{1} \otimes y_{2}}\right\} \\
& =\left\{u_{1} \in M_{1} \mid\left(\eta_{M_{1}}\left(u_{1}\right)\right)\left(u_{2}\right) \in U_{y_{1} \otimes y_{2}}\right\} \\
& =\left\{u_{1} \in M_{1} \mid \eta_{M_{1}}\left(u_{1}\right) \in\left[u_{2}, U_{y_{1} \otimes y_{2}}\right]\right\} \\
& =\eta_{M_{1}}^{-1}\left(\left[u_{2}, U_{y_{1} \otimes y_{2}}\right]\right) .
\end{aligned}
$$

Definition. Let $G: \underline{A} \rightarrow \underline{B}$ be a functor and let $M$ be an object of $\underline{B}$ a pair $(\mu, N)$, where $N$ is an object of $\underline{A}$ and $\mu: M \rightarrow G(N)$, is called a universal map for $M$ with respect to $G$ (or a $G$-universal map for $M$ ) provided that for each $N^{\prime}$ (object of $\underline{A}$ ) and each $f: M \rightarrow G\left(N^{\prime}\right)$, there is a unique $\underline{A}$-morphism $\bar{f}: N \rightarrow N^{\prime}$ such that the triangle commutes.
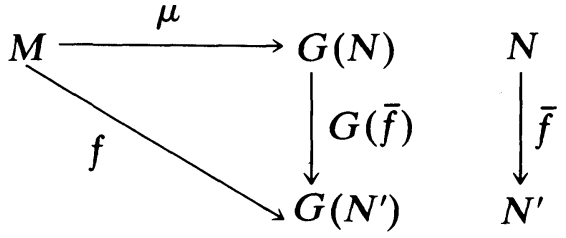

Notation. Given objects $\left(M_{1}, y_{\jmath}\right)$, for $j=1,2,3$, let $\operatorname{Bihom}\left(M_{1} \times M_{2}, M_{3}\right)$, denote the set of all bilinear maps $M_{1} \times M_{2} \rightarrow M_{3}$.

Proposition 28. The map $\theta$ : Bihom $\left(M_{1} \times M_{2}, M_{3}\right) \rightarrow$ $\operatorname{Hom}\left(M_{1}, \operatorname{Hom}\left(M_{2}, M_{3}\right)\right)$ given by, $\theta(\sigma)=\bar{\sigma}$, where $\left(\bar{\sigma}\left(u_{1}\right)\right)\left(u_{2}\right)=$ $\sigma\left(u_{1}, u_{2}\right)$, is a bijection.

THEOREM 29. Let $a \in \operatorname{Bihom}\left(M_{1} \times M_{2}, M_{3}\right)$. Then there exists $a$ unique $\quad \overline{\bar{\sigma}} \in \operatorname{Hom}\left(M_{1} \otimes M_{2}, M_{3}\right) \quad$ such that $\overline{\bar{\sigma}} \psi=\sigma \quad$ [where 
$\psi: M_{1} \times M_{2} \rightarrow M_{1} \otimes M_{2}$ is the canonical map, $\left(m_{1}, m_{2}\right) \rightarrow m_{1} \otimes m_{2}$; i.e., $\left.\theta(\psi)=\eta_{M_{1}}\right]$.

Proof.

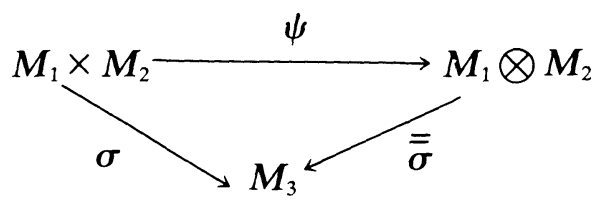

To complete the first diagram with a morphism $\overline{\bar{\sigma}}$, we make use of the fact that, by Theorem 27.3 [14] $\left(\eta_{M_{1}}, M_{1} \otimes M_{2}\right)$ is a universal map for $M_{1}$ with respect to $\operatorname{Hom}\left(M_{2}, \ldots\right)$.
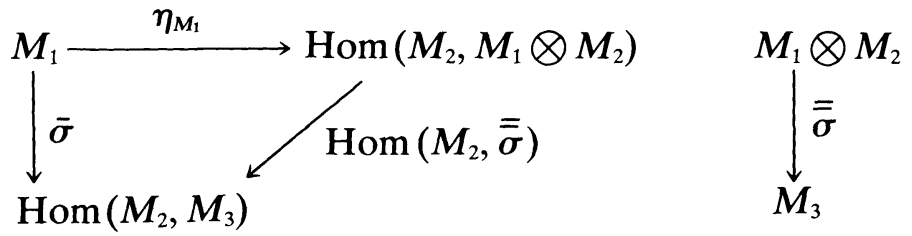

Thus, there exists a unique $\overline{\bar{\sigma}} \in \operatorname{Hom}\left(M_{1} \otimes M_{2}, M_{3}\right)$ such that $\bar{\sigma}=$ $\operatorname{Hom}\left(M_{2}, \overline{\bar{\sigma}}\right) \eta_{M_{1}}$ i.e., $\bar{\sigma}=\overline{\bar{\sigma}} \eta_{M_{1}}$. Note that $\overline{\bar{\sigma}}$ makes the first diagram commute.

Proposition 30. $M_{1} \otimes M_{2}=\left\{m_{1} \otimes m_{2} \mid m_{1} \in M_{1}, \quad m_{2} \in M_{2}\right\}$ and $y_{1} \otimes y_{2}$ is the closure operation coinduced on $M_{1} \otimes M_{2}$ by the family,

$$
F=\left\{\eta_{M_{1}}\left(m_{1}\right) \mid m_{1} \in M_{1}\right\} \cup\left\{\eta_{M_{2}}\left(m_{2}\right) \mid m_{2} \in M_{2}\right\} \text {. }
$$

Proof. Let $M=\left\{m_{1} \otimes m_{2} \mid \quad m_{1} \in M_{1}, \quad m_{2} \in M_{2}\right\}$. Then $M \subseteq$ $M_{1} \otimes M_{2}$. Although $F$ is not a covering family, we can form the coinduced closure, $y$ as follows: Let $Q_{1}=\left\{U \subseteq M \mid\left(\eta_{M_{1}}\left(m_{1}\right)^{-1}(U)\right)_{y_{2}}=\right.$ $\left.\eta_{M_{1}}\left(m_{1}\right)^{-1}(U) \quad \forall m_{1} \in M_{1}\right\} \cap\left\{U \subseteq M \mid \quad\left(\eta_{M_{2}}\left(m_{2}\right)^{-1}(U)\right)_{y_{1}}=\eta_{M_{2}}\left(m_{2}\right)^{-1}(U)\right.$ $\left.\forall m_{2} \in M_{2}\right\}$. Let $Q=\left\{U \in Q_{1} \mid \quad\left(U:\left(u_{1} \otimes u_{2}\right)\right)_{x}=U:\left(u_{1} \otimes u_{2}\right)\right.$, $\left.\forall u_{1} \otimes u_{2} \in M\right\}$ and note that $Q_{1}=Q$. For each $V \subseteq M$, let $V_{y}=$ $\cap\{U \in Q \mid V \subseteq U\}$. Then $(M, y)$ is an object of $M S$ and $y$ is the finest closure system on $M$ which permits all the $S$-maps in $F$ to be morphisms into $M$. Define $\xi: M_{1} \times M_{2} \rightarrow M$ by the rule: $\xi\left(m_{1}, m_{2}\right)=$ $m_{1} \otimes m_{2}$. Then $\xi$ is bilinear and surjective.

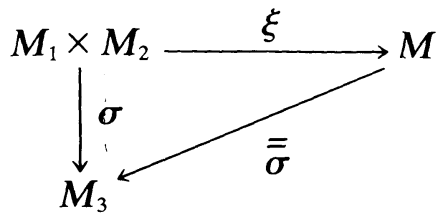


Let $\sigma: M_{1} \times M_{2} \rightarrow M_{3}$ be a bilinear map. Define $\overline{\bar{\sigma}}: M \rightarrow M_{3}$ by the rule: $\overline{\bar{\sigma}}\left(m_{1} \otimes m_{2}\right)=\sigma\left(m_{1}, m_{2}\right)$. Then $\overline{\bar{\sigma}}$ is a morphism and the diagram commutes. In fact, $\overline{\bar{\sigma}}$ is the identity morphism, $m_{1} \otimes m_{2} \rightarrow m_{1} \otimes m_{2}$ and, by Theorem 29, its inverse is also a morphism; hence, $M_{1} \otimes M_{2}=M$ and $y_{1} \otimes y_{2}=y$.

Proposition 31. For any objects $\left(M_{1}, y_{1}\right),\left(M_{2}, y_{2}\right)$ in $M S$, $M_{1} \otimes M_{2} \cong M_{2} \otimes M_{1}$.

Proposition 32. For any object $(M, y)$ of $M S, \quad S \otimes M \cong M \cong$ $M \otimes S$.

Proof. Let $\mu: S \otimes M \rightarrow M$ be the map given by $\mu(s \otimes m)=$ sm. Note that $\mu=\eta_{s}(1)^{-1} . \quad \mu$ is the required isomorphism.

Proposition 33. $\otimes$ is associative.

Proof. By Theorem 10 [17] it is enough to show that $\operatorname{Hom}\left(M_{1} \otimes M_{2}, M_{3}\right) \cong \operatorname{Hom}\left(M_{1}, \operatorname{Hom}\left(M_{2}, M_{3}\right)\right)$. By Theorem 27.9 [14], the adjoint situation, $(\eta, \delta):-\otimes M \dashv \operatorname{Hom}(M,-)$, gives a bijection $\alpha: \operatorname{Hom}\left(M_{1} \otimes M_{2}, M_{3}\right) \rightarrow \operatorname{Hom}\left(M_{1}, \operatorname{Hom}\left(M_{2}, M_{3}\right)\right)$ defined by the rule, $\left(\alpha(f)\left(m_{1}\right)\right)\left(m_{2}\right)=f\left(m_{1} \otimes m_{2}\right)$, for all $f \in \operatorname{Hom}\left(M_{1} \otimes M_{2}, M_{3}\right) . \alpha$ is the required isomorphism since, for all $m_{1} \otimes m_{2} \in M_{1} \otimes M_{2}$ and all $U_{y_{3}} \subseteq M_{3}$, $\alpha\left(\left[m_{1} \otimes m_{2}, U_{y_{3}}\right]\right)=\left[m_{1},\left[m_{2}, U_{y_{3}}\right]\right]$.

Proposition 34. _ $\otimes M$ preserves colimits. In particular, _ $\otimes M$ preserves coproducts.

Proposition 35. Let $\varphi \in \operatorname{Hom}\left(M_{1}, M_{2}\right)$. Then, for any object $(M, y) \quad$ in $M S \quad \varphi \otimes M: M_{1} \otimes M \rightarrow M_{2} \otimes M$ is the map, $u_{1} \otimes u \rightarrow \varphi\left(u_{1}\right) \otimes u$.

Proof.

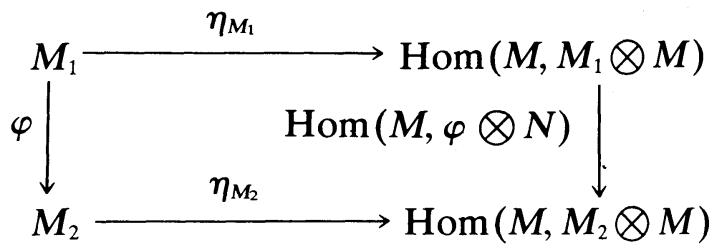

The adjoint situation $(\eta, \delta):_{-} \otimes M \dashv \operatorname{Hom}\left(M,{ }_{-}\right)$makes the diagram commute for each object $M$. Thus, for each $m_{1} \in M_{1}$, $\operatorname{Hom}(M, \varphi \otimes M)\left(\eta_{M_{1}}\left(m_{1}\right)\right)=\eta_{M_{2}}\left(\varphi\left(m_{1}\right)\right)$; i.e., for all $m \in M$, 


$$
\begin{aligned}
(\varphi \otimes M)\left(\eta_{M_{1}}\left(m_{1}\right)\right)(m) & =(\varphi \otimes M)\left(m_{1} \otimes m\right) \\
& =\eta_{M_{2}}\left(\varphi\left(m_{1}\right)\right)(m)=\varphi\left(m_{1}\right) \otimes m .
\end{aligned}
$$

Notation. $\quad \varphi \otimes 1$ will sometimes be written instead of $\varphi \otimes M$ in cases where no confusion will result.

Proposition 36. For any object $(M, y)$, the functor $-\otimes M$ preserves epimorphisms.

Definition. An object $(m, y)$ is Flat if the functor $-\otimes M$ preserves monomorphisms.

Proposition 37. S is a flat object of MS.

Proposition 38. Let $\left\{\left(M_{j}, y_{j}\right) \mid j \in J\right\}$ be a family of objects of MS. Then $\left(\Sigma M_{j}, \Sigma y_{j}\right)$ is flat iff $\left(M_{j}, y_{j}\right)$ is flat for each $j \in J$.

Proposition 39. Every projective object of MS is flat.

\section{Restriction and extension of scalars.}

Remarks. Let $\varphi:(S, x) \rightarrow\left(S^{\prime}, x^{\prime}\right)$ be a morphism of ideal systems; i.e., $\varphi(s t)=\varphi(s) \varphi(t)$, for all $s, t \in S$, and $\varphi\left(A_{x}\right) \subseteq(\varphi(A))_{x}$, for all $A \subseteq S$. Then any object $\left(M^{\prime}, y^{\prime}\right)$ of $M S^{\prime}$ can be considered as an object of $M S$ in the following manner: for each $s \in S, u^{\prime} \in M^{\prime}$, define $s u^{\prime}=$ $\varphi(s) u^{\prime}$. It is easy to verify that, with this $S$-set structure, $\left(M^{\prime}, y^{\prime}\right)$ is an object of $M S$ (the closure system $y^{\prime}$ does not change). This process is usually referred to as restriction of scalars. Let $\xi^{\prime} \in \operatorname{Hom}_{s^{\prime}}\left(M_{1}^{\prime}, M_{2}^{\prime}\right)$. If we restrict scalars as described above, we can consider both objects $M_{1}^{\prime}$ and $M_{2}^{\prime}$ as objects of $M S$ and then $\xi^{\prime}$ becomes an $S$-morphism with its $S$-map structure defined by the rule, $\xi^{\prime}\left(s u^{\prime}\right)=\xi^{\prime}\left(\varphi(s) u^{\prime}\right)$ for all $s \in S$.

Proposition 40. Let $\varphi:(S, x) \rightarrow\left(S^{\prime}, x^{\prime}\right)$ be a morphism of ideal systems. Then the process of restriction of scalars determines a faithful, covariant functor, $R_{\varphi}: M S^{\prime} \rightarrow M S$, which preserves monomorphisms and epimorphisms.

Definition. A functor which preserves monomorphisms and epimorphisms shall be called exact.

Proposition 41. Let $\varphi:(S, x) \rightarrow\left(S^{\prime}, x^{\prime}\right)$ be a morphism of ideal systems. Then the functor $R_{\varphi}: M S^{\prime} \rightarrow M S$ has a left adjoint $E_{\varphi}: M S \rightarrow M S^{\prime}$ given by the rule, $E_{\varphi}(M)=M \otimes R_{\varphi} S^{\prime}$ for all objects 
$(M, y)$ of $M S\left[E_{\varphi}(M)\right.$ is given $S^{\prime}$-set structure by defining for each $s^{\prime} \in S^{\prime}$ and each $\left.u \otimes t^{\prime} \in E_{\varphi}(M), s^{\prime}\left(u \otimes t^{\prime}\right)=u \otimes s^{\prime} t^{\prime}\right]$ and $E_{\varphi}(\delta)=\delta \otimes R_{\varphi} S^{\prime}$ for any morphism $\delta \in \operatorname{Hom}_{s}\left(M_{1}, M_{2}\right)$.

Proof. By Theorem 27.9 [14], it is sufficient to show that the two set-valued bifunctors, $\operatorname{hom}_{S^{\prime}}\left(E_{\varphi_{-}},{ }_{-}\right)$and $\operatorname{hom}_{s}\left({ }_{-}, R_{\varphi_{-}}\right)$are naturally isomorphic. Thus, let $(M, y)$ be an object of $M S$ and $\left(M^{\prime}, y^{\prime}\right)$ be an object of $M S^{\prime}$, and define $\beta: \operatorname{hom}\left(E_{\varphi} M, M^{\prime}\right) \rightarrow \operatorname{hom}\left(M, R_{\varphi} M^{\prime}\right)$ by the rule: $\beta(f)(m)=f\left(m \otimes 1^{\prime}\right) \forall m \in M$. Then $\beta$ is a bijection.

REMARK. The functor $E_{\varphi}: M S \rightarrow M S^{\prime}$ is usually referred to as extension of scalars.

Proposition 42. Let $\varphi:(S, x) \rightarrow\left(S^{\prime}, x^{\prime}\right)$ be a morphism of ideal systems. Then the functor $R_{\varphi}: M S^{\prime} \rightarrow M S$ has a right adjoint $H_{\varphi}: M S \rightarrow M S^{\prime}$ given by the rule: $H_{\varphi}(M)=\operatorname{Hom}_{S}\left(R_{\varphi} S^{\prime}, M\right) \forall$ objects $(M, y)$ of $M S\left[H_{\varphi}(M)\right.$ becomes an object of $M S^{\prime}$ by defining for each $s^{\prime} \in S^{\prime}$ and each $\left.\sigma \in H_{\varphi}(M),\left(s^{\prime} \sigma\right)\left(t^{\prime}\right)=\sigma\left(s^{\prime} t^{\prime}\right) \forall t^{\prime} \in R_{\varphi} S^{\prime}\right]$ and $H_{\varphi}(\lambda)=$ $\operatorname{Hom}_{S}\left(R_{\varphi} S^{\prime}, \lambda\right) \forall \lambda \in \operatorname{Hom}_{S}\left(M_{1}, M_{2}\right)$.

Proof. By Theorem 27.9 [14], it is sufficient to show that the two set-valued bifunctors, $\operatorname{hom}_{s}\left(R_{\varphi_{-}},{ }_{-}\right)$and $\operatorname{hom}_{S^{\prime}}\left({ }_{-}, H_{\varphi_{-}}\right)$are naturally isomorphic. Thus, let $(M, y)$ be an object of $M S$ and $\left(M^{\prime}, y^{\prime}\right)$ be an object of $M S^{\prime}$, and define $\gamma: \operatorname{hom}\left(R_{\varphi} M^{\prime}, M\right) \rightarrow \operatorname{hom}\left(M^{\prime}, H_{\varphi} M\right)$ by the rule: $\left(\gamma(g)\left(m^{\prime}\right)\right)\left(s^{\prime}\right)=g\left(s^{\prime} m\right) \forall s^{\prime} \in S^{\prime}$ and $\forall g \in \operatorname{hom}\left(R_{\varphi} M^{\prime}, M\right)$. Then $\gamma$ is a bijection.

Remark. Let $\varphi:(S, x) \rightarrow\left(S^{\prime}, x^{\prime}\right)$ be a morphism of ideal systems. Then for any object $(M, y)$ of $M S$ and any object $\left(M^{\prime}, y^{\prime}\right)$ of $M S^{\prime}, M \otimes R_{\varphi} M^{\prime}$ may be regarded as an object of $M S^{\prime}$ if it is given $S^{\prime}$-set structure in the following manner: $s^{\prime}\left(m \otimes m^{\prime}\right)=m \otimes s^{\prime} m^{\prime} \forall s^{\prime} \in S^{\prime}$ and $\forall m \otimes m^{\prime} \in M \otimes R_{\varphi} M^{\prime}$.

Proposition 43.' Let $\varphi:(S, x) \rightarrow\left(S^{\prime}, x^{\prime}\right)$ be a morphism of ideal systems. Let $(M, y)$ be an object of $M S$ and let $\left(M^{\prime}, y^{\prime}\right)$ be an object of $M S^{\prime}$. Then (in $\left.M S\right) M \otimes R_{\varphi} M^{\prime} \cong R_{\varphi}\left(E_{\varphi} M \otimes^{\prime} M^{\prime}\right)$ [where $\otimes^{\prime}$ indicates that the tensor product is formed in $\left.M S^{\prime}\right]$.

Proof. Let $\alpha: M \times R_{\varphi} M^{\prime} \rightarrow R_{\varphi}\left(E_{\varphi} M \otimes^{\prime} M^{\prime}\right)$ be defined by the rule: $\alpha\left(u, u^{\prime}\right)=\left(u \otimes 1^{\prime}\right) \otimes^{\prime} u^{\prime}$. Then $\alpha$ is $S$-bilinear; hence, there exists an $S$-morphism $\bar{\alpha}: M \otimes R_{\varphi} M^{\prime} \rightarrow R_{\varphi}\left(E_{\varphi} M \otimes^{\prime} M^{\prime}\right)$ such that the diagram commutes; i.e., $\bar{\alpha}\left(m \otimes m^{\prime}\right)=\alpha\left(m, m^{\prime}\right)=\left(m \otimes 1^{\prime}\right) \otimes^{\prime} m^{\prime}[\psi$ is the canonical bilinear map]. Note $\bar{\alpha}\left(m \otimes s^{\prime} m^{\prime}\right)=\left(m \otimes s^{\prime}\right) \otimes^{\prime} m^{\prime}$. 


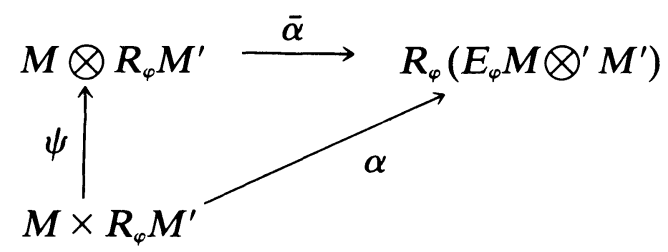

Let $\quad \hat{\alpha}: M \otimes R_{\varphi} M^{\prime} \rightarrow E_{\varphi} M \otimes^{\prime} M^{\prime}$ denote $\bar{\alpha}$ regarded as an $S^{\prime}$ morphism. Now, for each $m^{\prime} \in R_{\varphi} M^{\prime}$, define $\underline{m}^{\prime}: M \times$ $R_{\varphi} S^{\prime} \rightarrow M \otimes R_{\varphi} M^{\prime}$ by the rule: $\underline{m}^{\prime}\left(m, s^{\prime}\right)=m \otimes s^{\prime} m^{\prime}$. Then $\underline{m}^{\prime}$ is $S$-bilinear; hence, there exists an $S$-morphism $\underline{\hat{m}}^{\prime}: M \otimes R_{\varphi} S^{\prime} \rightarrow$ $M \otimes R_{\varphi} M^{\prime}$ such that $\underline{\hat{q}}^{\prime}\left(m \otimes s^{\prime}\right)=m \otimes s^{\prime} m^{\prime}$.

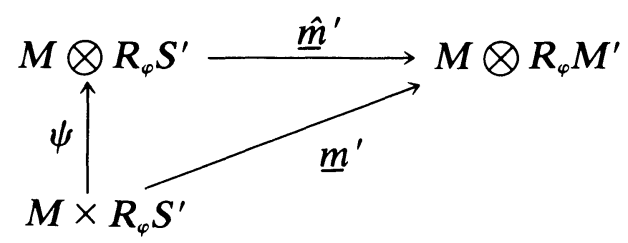

Actually, $\underline{\hat{m}}^{\prime}$ is an $S^{\prime}$-morphism with domain $E_{\varphi} M$ and codomain $M \otimes R_{\varphi} M^{\prime}$, the latter regarded as an object of $M S^{\prime}$. Let $\beta: E_{\varphi} M \times$ $M^{\prime} \rightarrow M \otimes R_{\varphi} M^{\prime}$ be defined by the rule: $\beta\left(m \otimes s^{\prime}, m^{\prime}\right)=$ $m \otimes s^{\prime} m^{\prime}$. Note that $\beta$ is well defined since, for each fixed $m^{\prime} \in R_{\varphi} M^{\prime}$, $\beta\left(\ldots, m^{\prime}\right)=\underline{\hat{m}}^{\prime}$ and, hence, does not depend upon the choice of representative of $m \otimes s^{\prime}$. Since $\beta$ is $S^{\prime}$-bilinear, it follows that there is an $S^{\prime}$-morphism $\hat{\beta}:\left(M \otimes R_{\varphi} S^{\prime}\right) \otimes M^{\prime} \rightarrow M \otimes R_{\varphi} M^{\prime} \quad$ such that $\hat{\beta}\left(\left(m \otimes s^{\prime}\right) \otimes^{\prime} m^{\prime}\right)=m \otimes s^{\prime} m^{\prime}$.

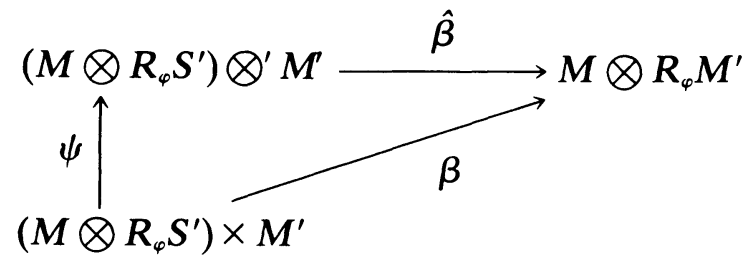

Thus, we have produced $S^{\prime}$-morphisms, $\hat{\alpha}: M \otimes R_{\varphi} M^{\prime} \rightarrow E_{\varphi} M \otimes^{\prime} M^{\prime}$ and $\hat{\beta}: E_{\varphi} M \otimes^{\prime} M^{\prime} \rightarrow M \otimes R_{\varphi} M^{\prime}$ which are inverses of one another; i.e., $M \otimes R_{\varphi} M^{\prime} \cong E_{\varphi} M \otimes M^{\prime}$ in $M S^{\prime}$. It follows that $M \otimes R_{\varphi} M^{\prime} \cong R_{\varphi}\left(E_{\varphi} M \otimes{ }^{\prime} M^{\prime}\right)$ in $M S$.

Proposition 44. Let $\varphi:(S, x) \rightarrow\left(S^{\prime}, x^{\prime}\right)$ be a morphism of ideal systems. Then the two MS-valued bifunctors, $-\otimes R_{\varphi_{-}}: M S \times$ $M S^{\prime} \rightarrow M S$ and $R_{\varphi}\left(E_{\varphi} \otimes_{-}\right): M S \times M S^{\prime} \rightarrow M S$ are naturally isomorphic.

Proposition 45. Let $\varphi:(S, x) \rightarrow\left(S^{\prime}, x^{\prime}\right)$ be a morphism of ideal systems. Suppose that $R_{\varphi} S^{\prime}$ is a flat object of MS. Then $R_{\varphi} M^{\prime}$ is flat in $M S$ for all flat objects $\left(M^{\prime}, y^{\prime}\right)$ in $M S^{\prime}$. 
Proof. The functor $-\otimes R_{\varphi} M^{\prime}: M S \rightarrow M S$ preserves monomorphisms whenever $\left(M^{\prime}, y^{\prime}\right)$ is a flat object of $M S^{\prime}$.

Proposition 46. Let $\varphi:(S, x) \rightarrow\left(S^{\prime}, x^{\prime}\right)$ be a morphism of ideal systems. Suppose that $(M, y)$ is a flat object of $M S$. Then $E_{\varphi} M$ is flat in $M S^{\prime}$.

Proof. Let $\xi: M_{1}^{\prime} \rightarrow M_{2}^{\prime}$ be an $S^{\prime}$-monomorphism. Then by Proposition $40, R_{\varphi} \xi: R_{\varphi} M_{1}^{\prime} \rightarrow R_{\varphi} M_{2}^{\prime}$ is an $S$-monomorphism; hence, since $M$ is flat in $M S, \quad M \otimes R_{\varphi} \xi: M \otimes R_{\varphi} M_{1}^{\prime} \rightarrow M \otimes R_{\varphi} M_{2}^{\prime}$ is an $S$ monomorphism. By Proposition 44, we have that $R_{\varphi}\left(E_{\varphi} M \otimes^{\prime} \xi\right)$ : $R_{\varphi}\left(E_{\varphi} M \otimes^{\prime} M_{1}^{\prime}\right) \rightarrow R_{\varphi}\left(E_{\varphi} M \otimes^{\prime} M_{2}^{\prime}\right)$ is an $S$-monomorphism; hence, $E_{\varphi} M \otimes^{\prime} \xi: E_{\varphi} M \otimes^{\prime} M_{1}^{\prime} \rightarrow E_{\varphi} M \otimes^{\prime} M_{2}^{\prime}$ is an $S^{\prime}$-monomorphism (since $R_{\varphi}$ is faithful).

\section{Monads and algebras in $M\{0,1\}$.}

Notation. We shall denote the category $M\{0,1\}$, simply, $\mathfrak{B}$. For any ideal system, $(S, x), \tau:\{0,1\} \rightarrow S$ will denote the map, $\tau(0)=0$, $\tau(1)=1$. Clearly $\tau$ is a morphism of ideal systems $[\{0,1\}$ is given the obvious ( $s$-system) closure system]. In the sequel we will denote $R_{\tau} S$, simply, $S$.

THEOREM 47. For any ideal system $(S, x), \bar{K}_{s}=\left(K_{s}, \eta, \mu\right)$ is a monad in $\mathfrak{B}$, where $K_{s}: \mathfrak{B} \rightarrow \mathfrak{B}$ is the functor, $-\otimes S$, and $\eta: 1_{\mathfrak{B}} \rightarrow K_{s}$ is the natural transformation given by, $\eta_{M}(m)=m \otimes 1$, and $\mu: K_{s} K_{s} \rightarrow K_{s}$ is the natural transformation, $\mu_{M}:(M \otimes S) \otimes S \rightarrow M \otimes S$ given by $\mu_{M}((m \otimes s) \otimes t)=m \otimes s t$.

Proof. The "unit," $\eta$, and the "multiplication," $\mu$, make the following diagrams commute:

where
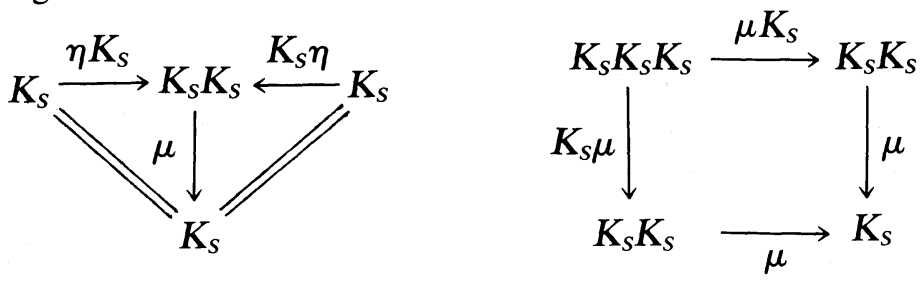

$$
\begin{aligned}
& \left(\eta K_{S}\right)_{M}=\eta_{M \otimes S}: M \otimes S \rightarrow(M \otimes S) \otimes S \\
& \left(K_{s} \eta\right)_{M}=\eta_{M} \otimes 1: M \otimes S \rightarrow(M \otimes S) \otimes S \\
& \left(\mu K_{S}\right)_{M}=\mu_{M \otimes S}:((M \otimes S) \otimes S) \otimes S \rightarrow(M \otimes S) \otimes S \\
& \left(K_{S} \mu\right)_{M}=\mu_{M} \otimes 1:((M \otimes S) \otimes S) \otimes S \rightarrow(M \otimes S) \otimes S .
\end{aligned}
$$


THEOREM 48. For any ideal system, $(S, x), \bar{H}_{s}=\left(H_{s}, \epsilon, \delta\right)$ is a comonad in $\mathfrak{B}$, where $H_{S}: \mathfrak{B} \rightarrow \mathfrak{B}$ is the functor, $\operatorname{Hom}\left(S,{ }_{-}\right)$, and $\epsilon: H_{S} \rightarrow 1_{\mathfrak{B}}$ is the natural transformation, $\epsilon_{M}: \operatorname{Hom}(S, M) \rightarrow M$, given by, $\epsilon_{M}(f)=f(1)$, and $\delta: H_{S} \rightarrow H_{S} H_{S}$ is the natural transformation, $\delta_{M}: \operatorname{Hom}(S, M) \rightarrow \operatorname{Hom}(S, \operatorname{Hom}(S, M))$, given by, $\left(\delta_{M}(f)(s)\right)(t)=(s f)(t)$ for all $t \in S$.

Proof. One must verify here that the following diagrams commute:
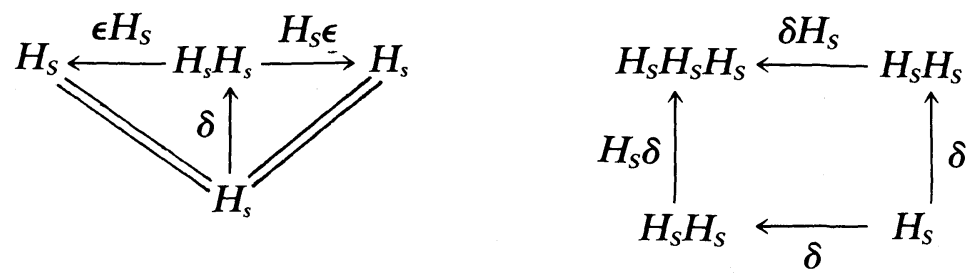

where

$$
\begin{aligned}
&\left(\epsilon H_{S}\right)_{M}= \epsilon_{\operatorname{Hom}(S, M)}: \operatorname{Hom}(S, \operatorname{Hom}(S, M)) \rightarrow \operatorname{Hom}(S, M) \\
&\left(H_{S} \epsilon\right)_{M}= \operatorname{Hom}\left(S, \epsilon_{M}\right): \operatorname{Hom}(S, \operatorname{Hom}(S, M)) \rightarrow \operatorname{Hom}(S, M) \\
&\left(\delta H_{S}\right)_{M}= \delta_{\operatorname{Hom}(S, M)}: \operatorname{Hom}(S, \operatorname{Hom}(S, M)) \\
& \rightarrow \operatorname{Hom}(S, \operatorname{Hom}(S, \operatorname{Hom}(S, M))) \\
&\left(H_{S} \delta\right)_{M}= \operatorname{Hom}\left(S, \delta_{M}\right): \operatorname{Hom}(S, \operatorname{Hom}(S, M)) \\
& \rightarrow \operatorname{Hom}(S, \operatorname{Hom}(S, \operatorname{Hom}(S, M)))
\end{aligned}
$$

REMARKs. Let $(S, x)$ be an ideal system and let $\mathfrak{B}^{s}$ denote the category of $\bar{K}_{S}$-algebras. Let $G: M S \rightarrow \mathfrak{B}^{s}$ be defined as follows: For each object $(M, y)$ of $M S, G(M)=\left\langle R_{r} M, h\right\rangle$, where $h: R_{\tau} M \otimes S \rightarrow R_{\tau} M$ is the $\mathfrak{B}$-morphism $m \otimes s \rightarrow s m$. For each $S$-morphism $f: M \rightarrow M^{\prime}$, $G(f)=R_{\tau} f: R_{\tau} M \rightarrow R_{\tau} M^{\prime}$. Then $G(f)$ is a $\mathfrak{B}^{s}$-morphism and, hence, $G$ is a (covariant) functor.

Now let $F: \mathfrak{B}^{s} \rightarrow M S$ be defined as follows: For each object $\langle M, h\rangle$ of $\mathfrak{B}^{s}$ (where $(M, y)$ is an object of $\left.\mathfrak{B}\right), F(\langle M, h\rangle)=(\bar{M}, y)$, where $\bar{M}=M$, equipped with the $S$-multiplication, $s m=h(s \otimes m)$ for all $s \in S, m \in M$. For any $\mathfrak{B}^{s}$-morphism $g:\langle M, h\rangle \rightarrow\left\langle M^{\prime}, h^{\prime}\right\rangle, F(g)=\bar{g}$, where $\bar{g}=g$, converted into an $S$-map by taking $\bar{g}(\mathrm{sm})=g(h(s \otimes m))$ for all $s \in S, m \in M$.

THEOREM 49. [Monadicity]. For any ideal system, $(S, x), \mathfrak{B}^{s}$ is isomorphic to MS.

Proof. With notation as in the remarks above we need only show 
that $F G=1_{M S}$ and $G F=1_{\mathfrak{B}^{s}}$. To show $F G=1_{M S}$ : since for any object $(M, y)$ of $M S, F G(M)=F\left(\left\langle R_{\tau} M, h\right\rangle\right)=\left(\overline{R_{\tau} M}, y\right)$, where $R_{\tau} M=R_{\tau} M$, endowed with an $S$-multiplication which is derived from the map $h$;i.e., $\left(\overline{R_{\tau} M}, y\right)=(M, y)$. For each object $\langle M, h\rangle$ of $\mathfrak{B}^{s}, \quad G F(\langle M, h\rangle)=$ $G((\bar{M}, y))=\left\langle R_{\tau} \bar{M}, \bar{h}\right\rangle$, where $\bar{h}: R_{\tau} \bar{M} \otimes S \rightarrow R_{\tau} \bar{M}$ is defined by the rule, $\bar{h}(m \otimes s)=s m=h(m \otimes s)$. Thus, $\bar{h}=h$, and, since it is clear that $R_{\tau} \bar{M}=M$, it follows that $G F(\langle M, h\rangle)=\langle M, h\rangle$.

Concluding remarks. The monads and comonads constructed above provide the tools with which resolutions and derived functors can be constructed which, in turn; lead to a (co)homology theory for $\mathfrak{B}$. The category of pointed topological spaces and basepoint preserving maps, $P T O P$, can be found in $\mathfrak{B}$. In fact, the inclusion functor PTOP $\rightarrow \mathfrak{B}$ is a full, faithful embedding.

\section{REFERENCES}

1. M. F. Atiyah, and I. G. MacDonald, Introduction to Commutative Algebra, Addison-Wesley, (1969).

2. Karl E. Aubert, Theory of $X$-ideals, Acta Mathematica, 107 (1962), 1-52.

3. —, Ideal systems and lattice theory I, Algebra Universalis, 1 (1971), No. 2, 204-213.

4. - Ideal Systems and Lattice Theory II, Tufts University (mimeographed notes) 1971.

5. Karl E. Aubert, and G. Gismarvik, Chinese Remainder Theorems in Ideal Systems, Tufts University (mimeographed notes) 1971.

6. Karl E. Aubert, Un Nouvel Axiome dans la Théorie, des Systèmes d'idéaux, C. R. Acad. Sci. Paris, t, 272 (1971), 422-425.

7. - Localisation Dans les Systèmes D'Idéaux, C. R. Acad. Sci. Paris, t, 272 (1971), 465-468.

8. Karl E. Aubert, and Erling R. Hansén, Systèmes De Modules, C. R. Acad. Sci. Paris, t, 272 (1971), 525-528.

9. —_ Additive Ideal Systems, J. Algebra, 18 (1971), 511-528.

10. M. Barr, and J. Beck, Homology and Standard Constructions. Seminar on Triples and Categorical Theory. Springer-Verlag, 80 (1969), 245-335.

11. Henri Cartan, and Samuel Eilenberg, Homological Algebra, Princeton University, (1956).

12. J. Duskin, $K(\pi, n)$-torsors and the interpretation of "triple" cohomology, Proc. Nat. Acad. Sci. USA, 71 (1974), 2554-2557.

13. Paul Ezust, Systems of Modules over Systems of Ideals, Thesis, Tuft University, 1975.

14. Horst Herrlich, and G. E. Strecker, Category Theory, Allyn and Bacon, (1973).

15. P. J. Hilton, and U. Stammbach, A Course in Homological Algebra, Springer-Verlag, (1970).

16. Peter Hilton, Lectures in Homological Algebra, Regional Conference Series in Mathematics \# 8. Am. Math. Soc., (1970).

17. G. M. Kelly, Tensor products in categories, J. Algebra, 2 (1965), 15-37.

18. F. E. J. Linton, Applied Functorial Semantics, Seminar on Triples and Categorical Theory, Springer-Verlag, 80 (1969), 53-74.

19. - An outline on Functorial Semantics, Seminar on Triples and Categorical Theory, Springer-Verlag, 80 (1969).

20. Saunders MacLane, Homology, Springer-Verlag, (1963). 
21. Categories for the Working Mathematician, Springer-Verlag, (1971).

22. Barry Mitchell, Theory of Categories, Academic Press, (1965).

23. Bodo Pareigis, Categories and Functors, Academic Press, (1970).

24. Bo Strenstrom, Flatness and Localization over Monoids, Math. Nachr., 48 (1971), 315-334.

25. F. Umer, On Cotriples and Andre (co)Homology, Their Relationship with Classical Homological Algebra, Seminar on Triples and Category Theory, Springer-Verlag, 80 (1969), 376-398.

Received June 30, 1975 and in revised form November 30, 1976. The results presented herein are drawn from the author's doctoral dissertation which was written at Tufts University under the direction of Professor George F. Leger.

SUFFOLK UNIVERSITY

BosTon, MA 02114 



\section{PACIFIC JOURNAL OF MATHEMATICS}

\section{EDITORS}

RichaRd ARENS (Managing Editor)

University of California

Los Angeles, CA 90024

\section{R. A. Beaumont}

University of Washington

Seattle, WA 98105

C. C. Moore

University of California

Berkeley, CA 94720
J. DugunduI

Department of Mathematics

University of Southern California

Los Angeles, CA 90007

R. Finn AND J. Milgram

Stanford University

Stanford, CA 94305

\section{ASSOCIATE EDITORS}
E. F. BECKENBACH
B. H. NeumanN
F. WOLF
K. YoshidA

\section{SUPPORTING INSTITUTIONS}

UNIVERSITY OF BRITISH COLUMBIA

CALIFORNIA INSTITUTE OF TECHNOLOGY

UNIVERSITY OF CALIFORNIA

MONTANA STATE UNIVERSITY

UNIVERSITY OF NEVADA

NEW MEXICO STATE UNIVERSITY

OREGON STATE UNIVERSITY

UNIVERSITY OF OREGON

OSAKA UNIVERSITY

\author{
UNIVERSITY OF SOUTHERN CALIFORNIA \\ STANFORD UNIVERSITY \\ UNIVERSITY OF HAWAII \\ UNIVERSITY OF TOKYO \\ UNIVERSITY OF UTAH \\ WASHINGTON STATE UNIVERSITY \\ UNIVERSITY OF WASHINGTON \\ AMERICAN MATHEMATICAL SOCIETY
}

The Supporting Institutions listed above contribute to the cost of publication of this Journal, but they are not owners or publishers and have no responsibility for its contents or policies.

Mathematical papers intended for publication in the Pacific Journal of Mathematics should be in typed form or offset-reproduced (not dittoed), double spaced with large margins. Underline Greek letters in red, German in green, and script in blue. The first paragraph or two must be capable of being used separately as a synopsis of the entire paper. Items of the bibliography should not be cited there unless absolutely necessary, in which case they must be identified by author and Journal, rather than by item number. Manuscripts, in duplicate, may be sent to any one of the four editors. Please classify according to the scheme of Math. Reviews, Index to Vol. 39. All other communications should be addressed to the managing editor, or Elaine Barth, University of California, Los Angeles, California, 90024.

100 reprints are provided free for each article, only if page charges have been substantially paid. Additional copies may be obtained at cost in multiples of 50 .

The Pacific Journal of Mathematics is issued monthly as of January 1966. Regular subscription rate: $\$ 72.00$ a year (6 Vols., 12 issues). Special rate: $\$ 36.00$ a year to individual members of supporting institutions.

Subscriptions, orders for back numbers, and changes of address should be sent to Pacific Journal of Mathematics, 103 Highland Boulevard, Berkeley, California, 94708.

PUBLISHED BY PACIFIC JOURNAL OF MATHEMATICS, A NON-PROFIT CORPORATION

Printed at Jerusalem Academic Press, POB 2390, Jerusalem, Israel. 


\section{Pacific Journal of Mathematics}

Vol. 68, No. 2

April, 1977

William Allen Adkins, Aldo Andreotti and John Vincent Leahy, An analogue of Oka's theorem for weakly normal complex spaces ........

Ann K. Boyle, M. G. Deshpande and Edmund H. Feller, On nonsingularly

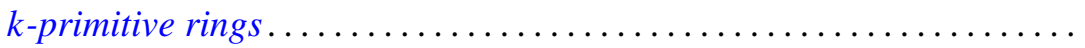

Rolando Basim Chuaqui, Measures invariant under a group of

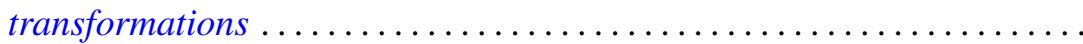

Wendell Dan Curtis and Forrest Miller, Gauge groups and classification of bundles with simple structural group .......................

Garret J. Etgen and Willie Taylor, The essential uniqueness of bounded nonoscillatory solutions of certain even order differential equations

Paul Ezust, On a representation theory for ideal systems

Richard Carl Gilbert, The deficiency index of a third order operator ........

John Norman Ginsburg, $S$-spaces in countably compact spaces using Ostaszewski's method.

Basil Gordon and S. P. Mohanty, On a theorem of Delaunay and some

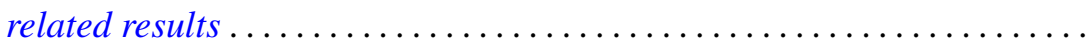

Douglas Lloyd Grant, Topological groups which satisfy an open mapping

theorem.

Charles Lemuel Hagopian, A characterization of solenoids

Kyong Taik Hahn, On completeness of the Bergman metric and its

subordinate metrics. II .

G. Hochschild and David Wheeler Wigner, Abstractly split group extensions.

Gary S. Itzkowitz, Inner invariant subspaces ...............

Jiang Luh and Mohan S. Putcha, A commutativity theorem for

non-associative algebras over a principal ideal domain.

Donald J. Newman and A. R. Reddy, Addendum to: "Rational approximation of $e^{-x}$ on the positive real axis".....

Akio Osada, On the distribution of a-points of a strongly annular function ....

Jeffrey Lynn Spielman, A characterization of the Gaussian distribution in a Hilbert space. .

Robert Moffatt Stephenson Jr., Symmetrizable-closed spaces ...

Peter George Trotter and Takayuki Tamura, Completely semisimple inverse $\Delta$-semigroups admitting principal series . . . . . . . .

Charles Irvin Vinsonhaler and William Jennings Wickless, Torsion free abelian groups quasi-projective over their endomorphism rings...

Frank Arvey Wattenberg, Topologies on the set of closed subsets ... 